\title{
Coerência entre editais de concurso público para professores e Diretrizes Curriculares dos cursos de Odontologia*
}

\author{
Raul Elton Araújo Borges**; Luiz Roberto Augusto Noro***
}

* Baseado no Trabalho de Conclusão de Curso “Avaliação da coerência entre os processos seletivos para docentes de cursos de odontologia em instituições federais brasileiras com as diretrizes curriculares nacionais", desenvolvido por Raul Elton Araújo Borges, disponível em: https://monografias.ufrn.br/jspui/bitstream/123456789/953/1/\%5B2014\%5 D\%20Avalia\%C3\%A7\%C3\%A3o\%20Da\%20Coer\%C3\%AAncia\%20Entr e\%200s\%20Processos\%20Seletivos\%20Para\%20Docentes.pdf

** Cirurgião-dentista, Universidade Federal do Rio Grande do Norte

*** Professor do Programa de Pós-Graduação em Saúde Coletiva e do Departamento de Odontologia da UFRN

Recebido em 24/09/2016. Aprovado em 20/10/2016.

\begin{abstract}
RESUMO
O presente estudo tem como objetivo traçar o perfil dos editais para seleção de professores de cursos de Odontologia em instituições públicas e analisar a coerência entre esses processos seletivos com as Diretrizes Curriculares Nacionais (DCN). A pesquisa foi desenvolvida utilizando os editais de concurso de universidades públicas no período de 2009 a 2014, com conceito 5 no Exame Nacional de Desempenho dos Estudantes (ENADE). Os resultados demonstram que a maioria dos processos seletivos é desenvolvida em quatro etapas: prova escrita, prova didática, defesa de memorial e análise de currículo. O perfil do processo seletivo encontrado remete à repetição de modelos tradicionais de ensino, com excessiva valorização da especialidade e fragmentação dos conhecimentos em disciplinas, não demonstrando coerência entre o que se recomenda nas DCN e os editais analisados nesse estudo. Para que o perfil dos egressos esteja em consonância com o preconizado pelas DCN, um desafio a ser enfrentado é pensar maneiras de reformular o processo seletivo para professores de Odontologia, assim como investir em processos de desenvolvimento docente permanente.

Descritores: Seleção de Pessoal. Educação Superior. Educação em Odontologia. Instituições de Ensino Superior.
\end{abstract}

\section{INTRODUÇÃO}

Em decorrência da implantação das Diretrizes Curriculares Nacionais (DCN) dos cursos de graduação, a qualidade do ensino superior na área da saúde passa a ser questionada, especialmente em função do não atendimento das demandas de saúde da população. As DCN não impõem um caráter único para os diversos cursos distribuídos no Brasil, mas definem uma base formadora sólida, que deve se dispor a ser 
constantemente buscada pelas instituições de educação superior ${ }^{1}$.

Para Feuerwerker ${ }^{2}$, as orientações de mudança na formação dos profissionais de saúde, norteadas pelas diretrizes curriculares, estão orientadas à superação de alguns problemas na formação profissional, marcada pela especialização e pela fragmentação dos conhecimentos, fatores muito relacionados ao perfil dos docentes, em especial na área da Odontologia. Assim, para melhorar a qualidade da educação superior torna-se necessário avaliar a forma como estes professores são selecionados, ou seja, em que bases se fundamentam os concursos públicos ou processos seletivos, para que os professores aprovados apresentem competências e habilidades adequadas e coerentes com os pressupostos previstos para a prática docente contemporânea ${ }^{3}$.

Os professores, por vezes, continuam formando seus alunos sem ação ou atividade que seja interdisciplinar, acabando por fragmentar a educação em temas isolados que estão desconectados e muitas vezes distantes com o que é preconizado e sinalizado nas $\mathrm{DCN}^{4,5}$. Deve-se salientar que o processo seletivo para docentes nas instituições públicas impacta diretamente a formação do profissional sinalizado nas DCN, uma vez que o professor desempenha papel fundamental na aprendizagem do aluno e no percurso profissional do egresso.

Faz-se necessária, portanto, uma reflexão sobre a forma como os professores ingressam nos cursos de Odontologia no Brasil, considerando os princípios do Sistema Único de Saúde (SUS) e as exigências do mercado de trabalho onde esse profissional irá atuar. Parte-se do pressuposto que estes docentes são peças fundamentais na formação dos cirurgiões-dentistas generalistas capazes de atuar em equipe na realidade social e nas necessidades de saúde bucal da população.

Diante disso, o presente estudo tem como objetivo traçar o perfil dos editais para seleção de professores de cursos de Odontologia em instituições públicas de ensino superior no Brasil e analisar a coerência entre esses processos seletivos com as Diretrizes Curriculares Nacionais para os cursos de Odontologia.

\section{MÉTODOS}

A presente pesquisa configura-se como estudo observacional descritivo, por meio de método quantitativo. O estudo foi desenvolvido a partir de dados secundários utilizando os editais para seleção de professores para o curso de Odontologia de instituições públicas federais brasileiras.

Para a identificação das unidades amostrais foram utilizados os indicadores de qualidade do ensino superior calculados pelo Instituto Nacional de Estudos e Pesquisas (INEP), com base nos resultados do ENADE 2010, no Conceito de Curso (CC) e o Conceito Preliminar de Curso (CPC).

Esses indicadores de qualidade são expressos em escala contínua de cinco níveis, em que os níveis iguais ou superiores a 3 indicam qualidade satisfatória. Eles servem como orientadores das avaliações in loco do ciclo avaliativo, sendo importantes instrumentos de avaliação da educação superior brasileira.

Para composição da amostra foram selecionados os cursos de Odontologia de instituições federais que apresentaram nota 5 no ENADE 2010 e igual ou superior a 4 tanto no no CC quanto no CPC. Dezessete cursos (quadro 1) cumpriram esses critérios, ou seja, apresentaram ótimo desempenho nestes componentes do Sistema Nacional de Avaliação da Educação Superior (SINAES).

A partir deste referencial foi realizada a busca de editais nos sítios web do Diário Oficial da União e das instituições selecionadas. Foram incluídos todos os editais abertos no período de 
2009 a 2014, de forma que a amostra final foi composta por 113 editais.

Os dados utilizados na pesquisa são de acesso público, não tendo sido, portanto, necessária a avaliação por Comitê de Ética em Pesquisa. A partir do download dos editais os dados foram coletados e armazenados em banco de dados. Foram coletadas as variáveis relativas à região brasileira, o tipo de componente curricular, à área de atuação e especialização exigida para o professor desenvolver suas atividades, titulação mínima necessária, regime de trabalho (RT), bem como relativas ao número e características das etapas previstas ao longo dos concursos. Os dados foram analisados por meio de estatística descritiva, a partir do programa Stata v.20 (StataCorp LP, College Station, TX, EUA).

Quadro 1: Cursos de Odontologia de universidades federais brasileiras segundo o Conceito de Curso (CC), Conceito Preliminar de Curso (CPC) e Exame Nacional de Desempenho dos Estudantes (ENADE), 2014.

\begin{tabular}{|lccc|}
\hline Nome da Instituição de Educação Superior & CC & CPC & ENADE \\
Universidade Federal de Sergipe & 4 & 4 & 5 \\
Universidade Federal do Amazonas & 4 & 4 & 5 \\
Universidade Federal de Uberlândia & 4 & 4 & 5 \\
Universidade Federal do Paraná & 4 & 4 & 5 \\
Universidade Federal do Espírito Santo & 4 & 4 & 5 \\
Universidade Federal de Minas Gerais & 4 & 4 & 5 \\
Universidade Federal de Juiz de Fora & 4 & 4 & 5 \\
Universidade Federal da Paraíba & 4 & 4 & 5 \\
Universidade Federal de Pernambuco & 4 & 4 & 5 \\
Universidade Federal de Goiás & 4 & 4 & 5 \\
Universidade Federal do Rio de Janeiro & 4 & 4 & 5 \\
Universidade Federal de Pelotas & 4 & 4 & 5 \\
Universidade Federal do Vale do Jequitinhonha e Mucuri & 4 & 4 & 5 \\
Universidade Federal do Mato Grosso do Sul & 4 & 4 & 5 \\
Universidade Federal do Ceará & 4 & 4 & 5 \\
Universidade Federal do Rio Grande do Norte & 5 & 4 & 5 \\
Universidade Federal do Rio Grande do Sul & 5 & 4 & 5 \\
\hline
\end{tabular}

Fonte: Instituto Nacional de Estudos e Pesquisas (INEP, 2014).

\section{RESULTADOS}

Observou-se que dos 113 editais disponíveis, $53(46,9 \%)$ eram de instituições localizadas na região Sudeste do Brasil, 39 $(34,5 \%)$ no Nordeste, $11(9,7 \%)$ no Sul, $8(7,1 \%)$ no Centro-Oeste e apenas $2(1,8 \%)$ na região Norte.

A relação entre os tipos de componentes curriculares e as áreas de atuação para as quais os editais foram publicados é verificada na tabela 1 . A maior parte dos editais ofertaram vagas para atuar em componente curricular do tipo disciplina $101(89,3 \%)$ enquanto apenas $9(8,0 \%)$ foram ofertados como módulos. Quanto a área de atuação, $86(76,1 \%)$ dos professores iriam desenvolver suas atividades em clínica odontológica e apenas $2(1,8 \%)$ trouxeram a exigência de atuação em mais de uma área.

Foi identificado um total de 23 especialidades, sendo a Saúde Coletiva a área de conhecimento com maior número de editais (14), correspondendo a $12,4 \%$ do total. As áreas de Periodontia e Prótese Dentária, ambas com 11, representaram cada uma $9,7 \%$ do total, enquanto 
a Cirurgia Buco-Maxilo-Facial, com 10 editais, correspondeu a $8,8 \%$.

Quanto à integração de conhecimentos, 101 editais $(89,3 \%)$ foram ofertados exigindo uma única especialização, ao mesmo tempo em que 12 editais $(10,6 \%)$ sinalizaram mais de uma área, sendo 8 o maior número de áreas de conhecimento exigidas em um mesmo edital para uma única vaga.

A grande maioria dos editais 108 (95,6\%) trouxe a exigência de o professor ter graduação em Odontologia. Quanto à titulação mínima necessária, $82(72,6 \%)$ exigiam doutorado, 20 $(17,6 \%)$ mestrado, $4(3,6 \%)$ especialização e 7 $(6,2 \%)$ apenas graduação.

Quando comparadas as variáveis titulação mínima exigida e região brasileira, observa-se que a exigência mínima dos níveis de especialização e graduação só foi encontrada em universidades localizadas no Norte e Nordeste brasileiros. Os resultados dessa distribuição estão expostos na tabela 2.

Tabela 1. Frequência e porcentagem do tipo de componente curricular em relação à característica da área de atuação do professor.

\begin{tabular}{|c|c|c|c|c|c|c|c|c|c|c|}
\hline \multirow{2}{*}{ Área } & \multicolumn{2}{|c|}{$\begin{array}{c}\text { Saúde } \\
\text { Coletiva }\end{array}$} & \multicolumn{2}{|c|}{ Clínica } & \multicolumn{2}{|c|}{ Básica } & \multicolumn{2}{|c|}{$\begin{array}{l}\text { Mais de } \\
\text { uma área }\end{array}$} & \multicolumn{2}{|c|}{ Total } \\
\hline & $\mathrm{n}$ & $\%$ & $\mathrm{n}$ & $\%$ & $\mathrm{n}$ & $\%$ & $\mathrm{n}$ & $\%$ & $\mathrm{n}$ & $\%$ \\
\hline Módulo & 0 & - & 8 & 7,1 & 1 & 0,9 & 0 & - & 9 & 8,0 \\
\hline Disciplina & 16 & 14,1 & 77 & 68,1 & 6 & 5,3 & 2 & 1,8 & 101 & 89,3 \\
\hline Estágio & 1 & 0,9 & 1 & 0,9 & 0 & - & 0 & - & 2 & 1,8 \\
\hline Outro & 0 & - & 0 & - & 1 & 0,9 & 0 & - & 1 & 0,9 \\
\hline Total & 17 & 15 & 86 & 76,1 & 8 & 7,1 & 2 & 1,8 & 113 & 100,0 \\
\hline
\end{tabular}

Tabela 2. Distribuição e porcentagem da titulação mínima exigida nos editais, de acordo com as regiões do país no qual se localiza a instituição, Brasil, 2014.

\begin{tabular}{l|cccccccccccc}
\hline \multirow{2}{*}{ Titulação } & \multicolumn{1}{c}{ Norte } & \multicolumn{1}{c}{ Nordeste } & \multicolumn{1}{c}{ Sudeste } & \multicolumn{2}{c}{ Sul } & \multicolumn{2}{c}{ Centro-Oeste } & \multicolumn{2}{c}{ Total } \\
& $\mathrm{n}$ & $\%$ & $\mathrm{n}$ & $\%$ & $\mathrm{n}$ & $\%$ & $\mathrm{n}$ & $\%$ & $\mathrm{n}$ & $\%$ & $\mathrm{n}$ & $\%$ \\
\hline Doutorado & - & 0 & 23 & 20,4 & 41 & 36,3 & 11 & 9,7 & 7 & 6,2 & 82 & 72,6 \\
Mestrado & - & 0 & 7 & 6,2 & 12 & 10,5 & - & 0 & 1 & 0,9 & 20 & 17,6 \\
Especialização & 2 & 1,8 & 2 & 1,8 & - & 0 & - & 0 & - & 0 & 4 & 3,6 \\
Graduação & - & 0 & 7 & 6,2 & - & 0 & - & 0 & - & 0 & 7 & 6,2 \\
Total & 2 & 1,8 & 39 & 34,6 & 53 & 46,8 & 11 & 9,7 & 8 & 7,1 & 113 & 100 \\
\hline
\end{tabular}

Em relação ao (RT) do professor, 84 dos editais $(74,3 \%)$ foram disponibilizados para o regime de dedicação exclusiva, $16(14,2 \%)$ no regime de 40 horas e $13(11,5 \%)$ no regime de 20 horas de trabalho semanais.

Quanto ao total de etapas previstas ao longo do concurso, o número variou entre duas e cinco etapas, sendo que 104 (92\%) se concentraram entre três e quatro etapas, sendo esta última a que obteve maior porcentagem (46,9\%). A realização de prova escrita foi observada em 91 editais $(80,5 \%)$, sendo 10 a média do número de tópicos ou temas exigidos em todos os formatos de provas: escrita, didática e prática. Destes tópicos, $54(59,3 \%)$ tiveram como características temas específicos da área de 
conhecimento exigida, $10 \quad(11 \%)$ temas integrados, ou seja, articulavam conteúdos de mais de uma área de conhecimento odontológico e $12(13,2 \%)$ sem identificação dos temas. Em 15 editais $(16,5 \%)$ essa informação não foi disponibilizada.

Em relação ao formato da prova escrita, 48 $(52,7 \%)$ aconteceram no formato de dissertação sobre um dos temas sorteados no momento da realização do concurso, $14(15,4 \%)$ no formato de múltipla escolha e dissertação sobre temas sorteados e em $12(13,2 \%)$ além de dissertação, o candidato à vaga também respondia a algumas perguntas sobre um dos temas sorteados. Em 17 $(18,7 \%)$ este formato não foi informado.

A realização de prova didática foi prevista em $95(84,1 \%)$ dos editais. A característica dos tópicos encontrados foi $63(66,3 \%)$ de temas específicos em uma única área de conhecimento, $12(12,6 \%)$ de temas integrados e em $20(21,1 \%)$ não foram identificados os temas. A aula expositiva foi o principal formato da prova didática, observado em $85(75,2 \%)$ dos editais.

Já a realização da prova prática foi prevista em 31 dos editais $(27,4 \%)$, sendo $20(64,5 \%)$ com tópicos em temas específicos e $11(35,5 \%)$ focados em situações-problema. $O$ principal formato dessa prova foi de atividade de desenvolvimento de habilidade manual no laboratório, presente em 17 (55,2\%) dos editais, $7(20,7 \%)$ no formato de atividade clínica na área de conhecimento $3(10,3 \%)$ com a simulação de casos clínicos (10,3\%). Em 2 (6,9\%) dos editais era prevista a realização de atividade clínica e laboratório, enquanto outros $2(6,9 \%)$ não informaram o formato.

$\mathrm{Na}$ totalidade dos editais foi prevista a análise do curriculum vitae dos candidatos, sendo que em $88(77,9 \%)$ essa etapa teve caráter classificatório, em $22 \quad(19,4 \%)$ caráter eliminatório e em $3(2,7 \%)$ classificatório ou eliminatório dependendo do departamento que solicitava o concurso.

A apresentação de memorial, plano de trabalho ou defesa do currículo foi exigida em menos da metade dos editais (45 - 39,8\%). Em 42 $(93,3 \%)$ destes havia previsão de normas para apresentação oral enquanto que $3(6,7 \%)$ não sinalizavam nenhuma norma para apresentação dos elementos que deveriam compor o memorial. Em $23(51,1 \%)$ a apresentação do memorial teve caráter eliminatório, $18(40 \%)$ classificatório e em $4(8,9 \%)$ classificatório ou eliminatório dependendo do departamento que solicitava do concurso.

A realização de entrevista com o candidato foi prevista em $18(15,9 \%)$ dos processos seletivos.

\section{DISCUSSÃO}

O Conselho Federal de Odontologia $^{6}$ revela que os 220 cursos de Odontologia de instituições públicas e privadas existentes no Brasil se concentram na região Sudeste (96, $40 \%$ ), com 43 instituições (20\%) na região Nordeste, 40 (18\%) na região Sul, 22 (10\%) na região Norte e 19 (9\%) no Centro-Oeste. Devido a essa distribuição desigual, o maior número de unidades formadoras de graduação e pósgraduação se localiza no Sudeste brasileiro.

As DCN para os cursos de Odontologia preconizam, na formação de cirurgiões-dentistas, um perfil de atuação profissional com formação generalista, humanista, crítica e reflexiva, para atuar em todos os níveis de atenção à saúde, com base no rigor técnico e científico ${ }^{7}$. A instituição dessas diretrizes vem desencadeando transformações em vários cursos de Odontologia do país.

Nesse sentido, há expectativas com relação à operacionalização das mudanças pretendidas, prevendo-se impacto concreto no perfil dos futuros cirurgiões-dentistas, no que diz respeito às suas qualificações para atuar adequadamente no âmbito do SUS. Estas mudanças estão fortemente vinculadas ao perfil dos docentes, uma vez que são esses os responsáveis pela formação profissional.

Observou-se nesse estudo que o perfil dos docentes que ocupam lugares nos cursos de graduação em Odontologia das instituições federais incluídas neste estudo tem se caracterizado pela excelência técnico-científica, uma vez que as vagas são ocupadas na quase totalidade por doutores e mestres nas diversas especialidades odontológicas. Em estudo 
realizado por Secco e Pereira ${ }^{9}$ com coordenadores dos cursos de Odontologia, a titulação acadêmica e o currículo foram apontados, por unanimidade, como muito valorizados e vistos, pela maioria dos coordenadores, como diferenciais da qualidade docente, o que corrobora com achados do presente estudo, em que a maioria dos editais exige o doutorado como titulação mínima necessária para concorrer ao processo seletivo.

Outro fator importante revelado no estudo foi que a maioria dos editais ofertaram vagas para atuação de professores em componente curricular do tipo disciplina, enquanto o tipo módulo - no qual há integração de saberes em diversas áreas de conhecimentos - aparece, ainda, como uma estratégia pouco utilizada por essas instituições formadoras. A fragmentação em disciplinas dificulta a perspectiva da formação de um generalista, ao centrar o ensino em uma perspectiva tecnicista e procedimental, inviabilizando a integração de conteúdos e a atuação interdisciplinar ${ }^{10}$. Em Odontologia, uma profissão historicamente marcada pela habilidade motora e procedimentos "artesanais", características privilegiadas nos currículos tradicionais, a interdisciplinaridade constitui uma alternativa fundamental para que áreas de especialidades delimitadas e segmentadas sejam articuladas em campos de conhecimento e produzam novas possibilidades e caminhos para a aprendizagem e formação em saúde.

Confirmando esta perspectiva, Toassi et al. ${ }^{11}$ relatam que o docente de Odontologia se caracteriza como especialista no seu campo de conhecimento e este é, inclusive, um critério essencial para sua seleção e contratação; porém não é um educador que domina a área educacional e pedagógica. O domínio do conteúdo e o sucesso da prática profissional são, comumente, considerados suficientes para o exercício da docência, contribuindo para que a valorização da formação docente no contexto da pós-graduação brasileira acabe sendo colocada em segundo plano em relação à formação do professor pesquisador ${ }^{12}$.

Ao identificar-se o perfil dos professores em instituições de educação superior no Brasil observam-se diferentes identidades. Existe uma variedade de docentes com formação didática obtida em cursos de licenciatura, geralmente vinculados a cursos nas áreas de Ciências Humanas e Sociais. Outros professores, em especial na área de Ciências da Saúde e Exatas, transportam automaticamente sua experiência profissional para a sala de aula, uma vez que a pós-graduação, em geral, pouco se apropria da perspectiva da formação pedagógica. Nessa conformação, na maioria das vezes, o definidor da seleção do docente é a competência científica $^{13}$.

O alto nível de especialização em uma única área de conhecimento exigido durante os processos seletivos reforçam a tendência de se formar "especialistas" ao invés de profissionais generalistas, uma vez que mestres e doutores com frequência não conseguem articular com sucesso seu campo de conhecimento a outros, em especial com o SUS. Torna-se imperativo, portanto, adequar o projeto pedagógico, a abordagem didático-pedagógica e os cenários de prática propiciando uma análise crítica do contexto, permitindo que o espaço profissional da prestação de assistência à saúde nos cursos de graduação seja repensado ${ }^{14}$.

Anastasiou $^{15}$ sinaliza que, atualmente, muitas instituições de ensino superior têm se preocupado em integrar seus currículos, sem, no entanto, alterar a lógica dos mesmos. Se não houver investimento na contratação de professores adequados e coerentes com este projeto, torna-se inviável imaginar reforma curricular plena ${ }^{16}$. O fato de $101(89,3 \%)$ dos editais analisados neste estudo ofertarem vagas para atuar em componente curricular do tipo disciplina representa um grande desafio para se encarar a perspectiva de construção de currículos integrados. Outro elemento importante observado e que requer reflexão é o enfoque preferencial da atuação na clínica odontológica. Isso revela ainda a ênfase da prática odontológica assistencial em cada especialidade, a valorização do ensino centrado na técnica e voltado para a atenção curativa, ao passo que o preconizado pelas DCN é uma prática odontológica de atuação generalista, com integração dos 
conhecimentos, interdisciplinaridade, com enfoque na promoção de saúde e nas necessidades do serviço de saúde e da população.

Em relação ao RT do professor universitário Lima e Filho $^{16}$ encontraram resultados equivalentes aos do presente estudo, onde o RT mais presente na amostra foi o regime de dedicação exclusiva (DE), que correspondeu a $74,9 \%$, seguido de 40 horas $(17,6 \%)$, sendo minoria o RT de 20 horas $(7,5 \%)$. A dedicação exclusiva pressupõe que o professor tenha toda sua capacidade dedicada à universidade, permitindo que seu vínculo seja caracterizado pelo comprometimento com a instituição nas esferas de ensino, pesquisa e extensão, dando condições de aprofundamento científico, articulação com outras instituições e responsabilidade social, que permitam a formação de profissionais de saúde efetivamente envolvidos com as condições da população e na busca de alternativas inovadoras para solução dos problemas de saúde.

$\mathrm{Na}$ Odontologia, a qualidade do ensino está relacionada entre tantos fatores com a qualificação e educação permanente (tanto técnica quanto didático-pedagógica) dos professores. Dessa forma, após esses resultados é possível refletir que no regime de dedicação exclusiva os professores, formados na visão biomédica e acostumados a ministrar suas aulas dentro dessa mesma visão, devem passar por ações de desenvolvimento docente, na perspectiva da educação permanente após ingressarem na instituição, uma vez que, para a concretização da mudança da abordagem do ensino sinalizado nas DCN, deve haver uma mudança nos papéis dos sujeitos envolvidos na dinâmica de ensino-aprendizagem de forma continuada e permanente. Esta estratégia poderia ser facilitada caso o processo seletivo já contemplasse a perspectiva de se selecionar professores com perfil mais adequado ao preconizado nas DCN.

Considerando que a educação permanente constitui um espaço privilegiado de reflexão sobre a prática docente, vale ressaltar que estimula, em todos os cenários, o acolhimento e a problematização das vivências, e amplia a troca de experiências entre os participantes, favorecendo o desenvolvimento pessoal e do processo de trabalho ${ }^{17}$. No âmbito das possibilidades, as propostas de desenvolvimento docente em saúde que vêm sendo implementadas e sinalizadas para os cursos da saúde parecem concorrer para convergências importantes que levam a reflexão sobre a prática docente em âmbito nacional.

Mitre et al. ${ }^{18}$ consideram, ainda, que a graduação dura somente alguns anos, enquanto a atividade profissional deve permanecer por décadas e que os conhecimentos e competências vão se transformando velozmente tornando-se, portanto, essencial pensar em metodologias de ensino para uma prática de educação libertadora, na educação permanente de professores ativos que estejam sempre aptos "a aprender a aprender" ${ }^{19,20}$.

No presente estudo foi ainda possível observar que a maior parte dos processos seletivos em universidades federais consiste, em média, de quatro etapas, sendo as principais a realização de prova escrita, prova didática, prova prática e análise de currículo. A prova escrita ainda acontece na lógica de dissertação sobre temas de uma área específica de conhecimento; a prova prática avalia a habilidade manual do candidato em laboratório ou clínica e a prova didática realizada no formato de uma aula expositiva sobre um tema sorteado, dentro da área específica de conhecimento exigida no edital. Não foram observadas exigências em relação a construção de planos de ensino ou de aula, desenvolvimento de propostas metodológicas ativas de aprendizagem, definição de protocolos de aprendizagem ou temas relacionados a pedagogia, por exemplo.

Numa nova configuração pretendida para a educação universitária, Carabetta Júnior e Cury ${ }^{21}$ sinalizam que se torna necessário o investimento na formação do professor na aquisição de competências relacionadas a socialização e significação dos conteúdos em diferentes contextos e de sua articulação interdisciplinar, bem como o conhecimento pedagógico e dos processos de investigação que possibilitam o aperfeiçoamento da prática docente. Dessa 
forma, para uma tradução dessas competências acreditamos que o processo seletivo deveria avaliar o conhecimento do conteúdo referente à disciplina ministrada, mas principalmente sobre como os diferentes conteúdos devem ser trabalhados, quais as metodologias que serão utilizadas pelo professor em sala de aula ou fora dela antes do exercício do magistério.

$\mathrm{O}$ perfil de processo seletivo revelado nesse estudo remete à repetição de modelos tradicionais de ensino acompanhados pelo professor ao longo de sua própria formação acadêmica ou após ocuparem a vaga em alguma das instituições de ensino em Odontologia. Lazarin $^{22}$ coloca a aula expositiva, a avaliação por meio da prova escrita e atividades laboratoriais acerca dos conteúdos de determinado conhecimento como a mais tradicional das estratégias de ensinoaprendizagem, sendo a atividade mais empregada pelos professores nos cursos de Odontologia.

Para Freitas et al. ${ }^{23}$, a eficiência da aprendizagem nas universidades e na capacitação de profissionais é muito baixa quando são utilizados os métodos tradicionais, e ressalta a importância das mudanças didático-pedagógicas proporcionadas pelo professor que vise uma aprendizagem centrada no estudante, baseada em metodologias ativas, as quais permitem que o estudante possa trabalhar com problemas reais, assumindo responsabilidades crescentes e interagindo com a população e os profissionais de saúde das áreas afins.

Segundo Baltazar" "os elementos fundamentais para a reformulação do ofício de docente em Odontologia devem estar vinculados a processos metodológicos e saberes pedagógicos, filosóficos, politicos, humanísticos e também, mas não apenas, saberes técnicos e especializados de cada área". É fundamental, portanto, envolvimento institucional no oferecimento de programas de desenvolvimento docente permanente, que enfoquem a necessidade da mudança ${ }^{25}$, os quais podem ser inseridos estrategicamente durante os processos seletivos em curso para contratação de professores.
Para Noro et $\mathrm{al}^{26}$, o paradigma da educação centrada no aluno como sujeito ativo de sua aprendizagem, mediado por professor que respeita a autonomia do aluno e constrói junto com ele o aprendizado, ainda se configura como um dos maiores desafios para formação em Odontologia. Segundo sugere a literatura, os docentes devem ter maior qualificação, desenvolvimento constante e contínuo, e reflexão e prática em novas estratégias de ensino muito além do conteúdo disciplinar ensinado ${ }^{27}$. O novo papel docente exige que o professor assuma o processo como mediador da aprendizagem ativa do estudante, transforme a escola das práticas multi e pluridisciplinares numa escola de práticas inter e transdisciplinares e integradas à vida cotidiana, conheça e aplique estratégias e metodologias ativas de ensinar-aprender a pensar, a aprender, a cuidar e avaliar ${ }^{13}$.

Considerando essas premissas, seria essencial que o processo seletivo trabalhasse com estratégias que buscassem o desenvolvimento destas habilidades, numa fase em que o candidato à vaga está bastante envolvido com sua perspectiva de ingresso a carreira docente, o que permitiria que o professor ingressante estivesse mais sensível às atividades de desenvolvimento docente coerentes com estes requisitos.

O aumento de cursos de pós-graduação stricto sensu ao longo da última década sinaliza a busca por parte significativa de profissionais de saúde pela carreira docente. Distante da formação específica da área profissional, e mais ainda da área da educação, muitas vezes estes mestres e doutores transformam-se em professores com pouco embasamento quer nos princípios pedagógicos, quer no conhecimento dos princípios que norteiam a vida acadêmica. Acabam por fazer seu percurso docente a partir da repetição do que vivenciaram em sua formação ou a partir de demandas estabelecidas pela administração acadêmica da instituição na qual irão atuar. Estas, por sua vez, procurando minimizar tais deficiências, ofertam atividades de capacitação pedagógica, com as mais diversas nomenclaturas, visando inserção do professor no meio acadêmico. Entretanto, na maioria das vezes, essas iniciativas apresentam pouca 
proximidade com a área específica onde o professor irá atuar e, apesar de válidas, acabam por não se consolidar como uma política permanente de formação docente, uma vez que dependem da iniciativa do professor em participar desses momentos.

Parece óbvio, portanto, que o momento de ingresso do professor na docência universitária é estratégico para que possa assumir um compromisso efetivo com uma prática docente com base em abordagens pedagógicas contemporâneas, que viabilizem o protagonismo do aluno em seu processo formativo.

Outro fator que reforça esse momento estratégico é que, ao longo dos últimos anos observou-se uma disputa crescente para se ingressar em instituições públicas. Os candidatos às vagas dedicam várias horas de estudo para o concurso e se condicionam àquilo que o processo seletivo solicita. Assim, se o essencial para aprovação do professor no concurso é ministrar uma aula expositiva sobre determinado tema, é nisso que o candidato dedicará sua expertise. Se for exigida determinada especialização, é a partir dela que o professor fundamentará sua prática docente, o que o distancia da formação generalista.

Além disso, esse momento deve prever elementos nos quais fique clara a compreensão das DCN, em sua essência e princípios, por parte do ingressante, além de oportunizar atividades sobre como promover formação generalista tendo o SUS como cenário prioritário. Ou seja, para viabilizar o proposto pelas DCN é urgente pensar nos futuros professores, antes mesmo de eles ingressarem na Universidade.

\section{CONCLUSÃO}

Após a análise dos processos seletivos para docentes nos cursos de Odontologia de universidades federais brasileiras é notória ainda a incoerência que existe na forma de recrutamento dos professores com o que é sinalizado pelas DCN, pois o processo continua infundindo a ideia de formar profissionais "especialistas", remetendo à repetição de modelos tradicionais de ensino, com excessiva valorização da especialização e da fragmentação dos conhecimentos. É fator fundamental, portanto, repensar-se os processos seletivos para que esses sejam coerentes com a viabilização de projetos pedagógicos adequados às necessidades de saúde bucal da população e com os preceitos das DCN, considerando o papel estratégico desempenhado por um professor produtor de conhecimento, reorientando a Odontologia em benefício de toda a sociedade.

\section{ABSTRACT \\ Consistency between public position announcements for teachers and Curricular Guidelines for Dentistry courses}

The present study aims to draw the profile of position announcements for selecting Dental School teachers in federal public institutions and to analyze the consistency between these selective processes and Brazilian National Curriculum Guidelines (DCN). The research was conducted using data from schools that reached grade 5 in National Exam of Student Performance (ENADE) in the 2009-2014 assessment. The results showed that most selection processes are developed in four stages: written test, teaching test, memorial and curriculum analysis. The profile of the selective process found repeats the traditional teaching models, with excessive fragmentation of knowledge and specialization into disciplines. The position announcements are not consistent with the DCN's recommendations. A challenge to be faced in order to reach the graduates profile in line with DCN is to reshape the selection process for dental teachers, as well as invest in permanent teacher development processes

Descriptors: Personnel Selection. Education, Higher. Education, Dental. Higher Education Institutions.

\section{REFERÊNCIAS}

1. Freitas SFT, Calvo MCM, Lacerda JT. Saúde coletiva e novas diretrizes curriculares em odontologia: uma proposta para graduação. Trab Educ Saúde. 2012; 10(2):223-34.

2. Feuerwerker LCM. Educação dos profissionais de saúde hoje - problemas, 
desafios, perspectivas e as propostas do Ministério da Saúde. Rev ABENO. 2003; 3(1):24-7.

3. Goldwasser RS. A Prova Prática no Processo de Seleção do Concurso de Residência Médica. Rev Bras Educ Méd. 2006; 30(3):115-24.

4. Diógenes VCN, Souza GCA, Emiliano GBG, José Júnior FL, Suliano AA. Teaching-learning process in times of the Unifield Health System (SUS): training of faculty and dental surgeons in Brazil. Rev Odonto Ciênc. 2010; 25(1):92-6.

5. Forte FDS, Pessoa TRRF, Freitas CHSM, Pereira CAL, Carvalho Júnior PM. Reorientação na formação de cirurgiõesdentistas: o olhar dos preceptores sobre estágios supervisionados no Sistema Único de Saúde (SUS). Interface 2015; 19(supl.1) :831-43.

6. Conselho Federal de Odontologia (CFO). Distribuição de cursos de Odontologia /Brasil. [Acesso em 25 out. 2016]. Disponível em: http://cfo.org.br/wp-content/uploads /2009/10/quadro_estatistico_faculdade.pdf

7. Resolução CNE/CES no 3, de 20 de junho de 2014. Institui Diretrizes Curriculares Nacionais do Curso de Graduação em Medicina e dá outras providências. Diário Oficial da União. 23 Jun 2014.

8. Carvalho RB, Costa TBC, Gomes MJ, Santos KT, Guerra SMG. Formação docente em odontologia no Brasil: sugestões de mudanças após as diretrizes curriculares nacionais. Rev Bras Pesq Saúde. 2010; 12(4):39-44.

9. Secco LG, Pereira MLT. Concepções de qualidade de ensino dos coordenadores de graduação: uma análise dos cursos de odontologia do Estado de São Paulo. Interface Comun Saúde Educ. 2010;8(15) :313-30.

10. Gomes CS, Nadaes MR, Senna MMA, Gouvêa MV, Dias NM, Sirimarco PN. Perspectiva do ensino odontológico na ótica do discente da Faculdade de Odontologia da Universidade Federal Fluminense: novas diretrizes curriculares e perfil profissional.
Rev ABENO. 2012; 12(2):233-39.

11. Toassi RFC, Stobaus CD, Mosquera JJM, Moyses SJ. Currículo Integrado no ensino de Odontologia: novos sentidos para a formação na área da saúde. Interface Comun Saúde Educ. 2012; 16(41):529-42.

12. Batista NA. Desenvolvimento docente na área da saúde: uma análise. Trab Educ Saúde. 2005; 3(2):283-94.

13. Machado JLM, Machado VM, Vieira JE. Formação e seleção de docentes para currículos inovadores na graduação em saúde. Rev Bras Educ Méd. 2011; 35(3) :326-33.

14. Lampert JB, Perim GL, Aguilar-da-Silva RH, Stella RCR, Abdala IG, Costa NMSC. Mundo do trabalho no contexto da formação. Rev Bras Educ Méd. 2009; 33(1 Supl.1):3543.

15. Anastasiou LGC. Da visão de ciência à organização curricular. IN: Anastasiou, LGC. Processos de Aprendizagem na universidade: pressupostos para estratégias de trabalho em aula. Joinville: UNIVILLE 2005. p. 45-63.

16. Costa ICC. Os sete saberes necessários a educação do futuro e planejamento das ações de saúde: algumas reflexões e confluências. Rev ABENO. 2007;7(2):122-9.

17. Lazarin CA, Francischetti I. Educação Permanente: uma ferramenta para o desenvolvimento docente na graduação. Rev Bras Educ Méd. 2010;34(4):481-6.

18. Mitre SM, Siqueira-Batista R, Girardi-deMendonça JM, Morais-Pinto NMM, Meirelles CAB, Pinto-Porto $\mathrm{C}$ et al. Metodologias ativas de ensinoaprendizagem na formação profissional em saúde: debates atuais. Ciênc Saúde Coletiva. 2008;13(Supl 2):2133-44.

19. Freire P. Pedagogia da Autonomia: saberes necessários à prática educativa. São Paulo: Paz e Terra, 1997.

20. Morin E. Os sete saberes necessários á prática educativa. São Paulo: Cortez, 1999.

21. Carabetta Júnior V, Cury MCFS. A contribuição da coordenação pedagógica na escola de Medicina. Rev Bras Educ Méd. 
2007; 31(1):44-51.

22. Lazarrin HC, Nakama L, Cordoni JL. O papel do professor na percepção dos alunos de Odontologia. Saúde Soc. 2007; 16(1):90101.

23. Freitas VP, Carvalho RB, Gomes MJ, Figueiredo MC, Faustino-Silva DD. Mudança no processo ensino-aprendizagem nos cursos de graduação em odontologia com utilização de metodologias ativas de ensino e aprendizagem. RFO UPF. 2009; 4(2):163-67.

24. Baltazar MMM. Profissão, Docente de Odontologia: O desafio da Pós-Graduação na Formação de Professores. Trab Educ Saúde. 2010; 8(2):285-303.

25. Mendonça ET, Cotta RMM, Lelis VP, Carvalho Júnior PM. Paradigmas e tendências do ensino universitário: a metodologia da pesquisa-ação como estratégia de formação docente. Interface Comun Saúde Educ. 2015;19(53):373-86

26. Noro LRA, Farias-Santos BCS, Souza PHS, Pinheiro IAG, Borges REA, Nunes LMF et al. O professor (ainda) no centro de processo ensino-aprendizagem. Rev ABENO. 2015; 15(1):2-11.

27. Rêgo CI, Batista SH. Desenvolvimento Docente nos Cursos de Medicina: um Campo Fecundo. Rev Bras Educ Méd. 2012; 36(3):317-24.

Correspondência para:

Prof. Dr. Luiz Roberto Augusto Noro

e-mail: luiz_noro@hotmail.com

Departamento de Odontologia da UFRN Avenida Salgado Filho, 1787

59056-000 - Natal/RN 